\title{
Structures And Bandgaps Of Small Range Gallium Arsenide Nanocluster
}

\author{
Nor Muniroh Musa ${ }^{1, *}$, Ahmad Radzi Mat Isa², and Mohd Khalid Kasmin ${ }^{1}$ \\ ${ }^{1}$ Physics Department, Faculty of Science, Universiti Teknologi Malaysia, 81310 UTM Skudai, Johor, Malaysia \\ ${ }^{2}$ Ibnu Sina Institute for Fundamental Sciences Studies, Universiti Teknologi Malaysia, 81310 UTM Skudai, Johor, Malaysia \\ *Author to whom correspondence should be addressed; E-mail: muniroh_82@yahoo.com
}

Received: 28 October 2008

http://dx.doi.org/10.11113/mjfas.v4n2.54

\section{ABSTRACT}

There is currently a lot of excitement about Gallium Arsenide nanoclusters due in part to the prediction that smallenough GaxAsy clusters will act as "quantum dot" whose bandgaps can be tuned by varying the cluster size. Small range GaAs clusters $(x+y \leq 10)$ were studied by using first principles calculation where structural properties and bandgap were investigated. We obtained the results of bandgap which are larger than the simulated bandgap of bulk GaAs. In this paper, we discuss the effect of GaAs cluster size to the energy bandgap.

| Gallium Arsenide | Nanocluster | Quantum Dot | Band gap |

\section{Introduction}

Gallium Arsenide nanostructures are being a popular subject of research since last three decades because of its fascinating properties and potential for nanoelectronic devices which is the trend of miniaturization nowadays. Many studies showed that GaAs nanoclusters have a significant change of electronic properties from the bulk. This has been the favor in the fabrication of nano-electronic devices for example, optoelectronic devices and transistors. One of the devices drawing great attention is the Single-Electron Transistor (SET), which has the properties of low-power consumption and high-speed performance. SET consists of a quantum dot with sourcedrain electrodes as well as a gate electrode. There is currently a lot of effort being made to study the GaAs quantum dots based on the GaAs clusters for their electronic structures.

One of the phenomenons that make nanoclusters or quantum dots become crucial in nanodevices is quantum confinement. It can be observed when the dimensionality of a crystal decreases from bulk to nano dimensions $(\leq 10 \mathrm{~nm})$. During this size reduction, the electronic band of the crystal is gradually quantized starting from the band edges as a function of size reduction which consequently results in an increase in the band gap energy (Figure1)[1]. This quantum size effect also results in a blue-shift of energy gap with decreasing size. 

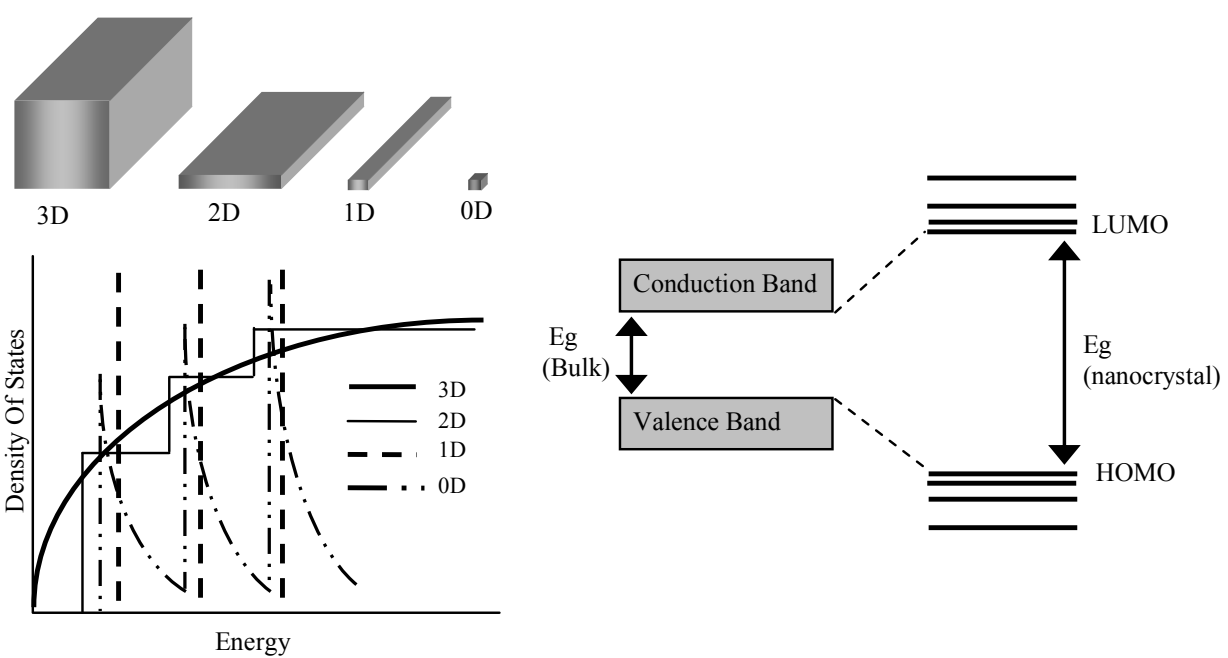

Figure 1: (a) Density of state for different dimension [1]. (b) Difference of electronic states in bulk semiconductor and a nanocrystal [2].

\section{Computational Details}

The calculations presented here are based on the density-functional theory (DFT) in the local-density approximation (LDA). We employ and implement the code of Vienna ab initio simulation package (VASP)[2-4], which uses plane wave basis set with generalized gradient approximation (GGA)[5] for exchange-correlation functional. The pseudopotentials are combined with the projected-augmented wave (PAW)[6] method which describes the interaction between ions and electrons. A conjugate-gradient (CG) [7] algorithm is used to optimize the atomic structures.

In order to simulate an isolated cluster, supercell approach is implemented. Supercell size of $1.6 \mathrm{~nm}$ was used to omit the interaction between the clusters and its periodic image in 3D. We use a cutoff energy of $250 \mathrm{eV}$ for the plane wave expansion to ensure precise results obtained. Gaussian smearing of $0.1 \mathrm{eV}$ was used in order to perform the density of states. Owing to the supercell approach, the cluster calculations are performed using the $\Gamma$ point only. Before the electronic structures simulations, the structure of the GaAs clusters is allowed to be optimized in the self consistent cycle. After the structure optimization, band structure calculations are performed.

\section{Results And Discussion}

Figure 2 shows the lowest-energy structures of each $\mathrm{Ga}_{\mathrm{x}} \mathrm{As}_{\mathrm{y}}(x+y \leq 10)$ which are also the most stable structure. Calculations find the symmetry group of $\mathrm{C}_{2 v}$ linear for GaAs, $\mathrm{C}_{2 \mathrm{v}}$ triangle for $\mathrm{Ga}_{1} \mathrm{As}_{2}, \mathrm{D}_{2 \mathrm{~h}}$ planar rhombus for $\mathrm{Ga}_{2} \mathrm{As}_{2}, \mathrm{C}_{2 \mathrm{v}}$ trigonal bipyramid for $\mathrm{Ga}_{2} \mathrm{As}_{3}, \mathrm{C}_{1}$ capped trigonal bipyramid for $\mathrm{Ga}_{3} \mathrm{As}_{3}, \mathrm{C}_{\mathrm{s}}$ capped prism for $\mathrm{Ga}_{3} \mathrm{As}_{4}, \mathrm{C}_{1}$ rhombic prism for $\mathrm{Ga}_{4} \mathrm{As}_{4}, \mathrm{C}_{\mathrm{s}}$ tricapped trigonal prism for $\mathrm{Ga}_{4} \mathrm{As}_{5}$ and $\mathrm{C}_{\mathrm{s}}$ tetracapped trigonal prism for $\mathrm{Ga}_{5} \mathrm{As}_{5}$. The structures of $\mathrm{Ga}_{\mathrm{x}} \mathrm{As}_{\mathrm{y}}(4 \leq x+y \leq 6)$ is built according to edge-capping mode while face-capping growth mode is preferred for $\mathrm{Ga}_{\mathrm{x}} \mathrm{As}_{\mathrm{y}}(7<x+y<10)$ [8]. 
The accuracy of the calculation is tested first for bulk Gallium Arsenide. For the zinc blende structure GaAs with lattice constant $5.653 \AA$ which is close to experimental lattice constant $5.632 \AA$, the band gap obtained from the simulation is $0.5453 \mathrm{eV}$ which is lower than the experimental result. This is because density functional is a ground state theory and thus it underestimated the band gap value.

Table 1 shows the energy versus bandgap (highest occupied molecular orbital - lowest unoccupied molecular orbitals gap) of Gallium Arsenide clusters with different number of atom. Overall, the bandgaps of Gallium Arsenide nanocluster $\mathrm{Ga}_{\mathrm{x}} \mathrm{As}_{\mathrm{y}}$ are larger than the bulk Gallium Arsenide. It shows that when GaAs reduces to nanosize, its properties will change where the bandgap will increase. According to quantum theory, the bandgap increases as the size decreases. This indicates the stronger confinement for the smaller sizes. However, Figure 3 shows the results that do not comply with the above theory. Instead, it changes unevenly up and down between $0.7370 \mathrm{eV}$ to $3.0956 \mathrm{eV}$. However, our results are in good agreement with those reported in literatures [10]. Among these nanoclusters $(x+y \leq 10), \mathrm{GaAs}$ is observed to posses the highest bandgap energy whilst $\mathrm{Ga}_{4} \mathrm{As}_{5} \mathrm{has}$ the lowest value.

We can say that when the size of the cluster is very small $(<1 \mathrm{~nm})$, the bandgap energy does not increase with the decreasing of size. However, with the widening of the bandgaps from bulk, small GaAs clusters are still showing their evolvement and difference from bulk as size reduction. The bandgap depends strongly on the number of atoms and atomic structure. For a structure with the same number of atom but different configuration, the bandgap might be different.

So, the results can be used to predict the performance of the SET. When the cluster between the source and drain becomes smaller in size, the energy gap increases which means the confinement effect is stronger. This can optimize the operating temperature of SET because of the relation $E>k T$, where $E$ is the energy similar to the energy spacing of quantum dot. Besides that, we can predict the I-V characteristic of SET by understanding the energy level of the quantum dot and that we can know how much energy should be applied to pass quantized electrons.

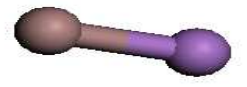

GaAs
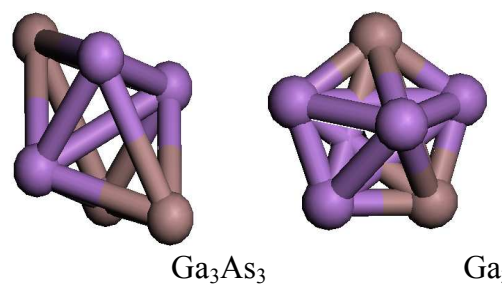

$\mathrm{Ga}_{3} \mathrm{As}_{4}$

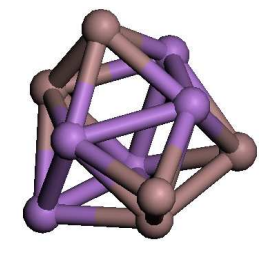

$\mathrm{Ga}_{4} \mathrm{As}_{4}$

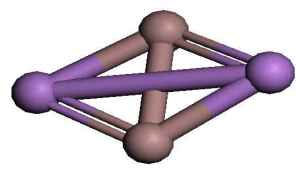

$\mathrm{Ga}_{2} \mathrm{As}_{2}$

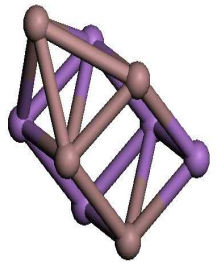

$\mathrm{Ga}_{4} \mathrm{As}_{5}$

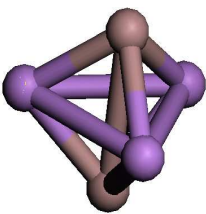

$\mathrm{Ga}_{2} \mathrm{As}_{3}$

Figure 2: Lowest energy structures of $\mathrm{Ga}_{\mathrm{x}} \mathrm{As}_{\mathrm{y}}$ 


\begin{tabular}{|c|c|c|}
\hline $\mathbf{G a}_{\mathbf{x}} \mathbf{A s} \mathbf{s}_{\mathbf{y}}$ & $\begin{array}{c}\text { Symmetry } \\
\text { Group }\end{array}$ & $\begin{array}{c}\text { Bandgap } \\
\text { energy (eV) }\end{array}$ \\
\hline $\mathrm{GaAs}$ & $\mathrm{C}_{2 \mathrm{v}}$ & 3.0956 \\
\hline $\mathrm{Ga}_{1} \mathrm{As}_{2}$ & $\mathrm{C}_{2 \mathrm{v}}$ & 0.8756 \\
\hline $\mathrm{Ga}_{2} \mathrm{As}_{2}$ & $\mathrm{D}_{2 \mathrm{~h}}$ & 1.4031 \\
\hline $\mathrm{Ga}_{2} \mathrm{As}_{3}$ & $\mathrm{C}_{2 \mathrm{v}}$ & 1.0918 \\
\hline $\mathrm{Ga}_{3} \mathrm{As}_{3}$ & $\mathrm{C}_{1}$ & 1.6689 \\
\hline $\mathrm{Ga}_{3} \mathrm{As}_{4}$ & $\mathrm{C}_{\mathrm{s}}$ & 1.5755 \\
\hline $\mathrm{Ga}_{4} \mathrm{As}_{4}$ & $\mathrm{C}_{\mathrm{i}}$ & 1.0062 \\
\hline $\mathrm{Ga}_{4} \mathrm{As}_{5}$ & $\mathrm{C}_{\mathrm{s}}$ & 0.7370 \\
\hline $\mathrm{Ga}_{5} \mathrm{As}_{5}$ & $\mathrm{C}_{\mathrm{s}}$ & 1.5898 \\
\hline
\end{tabular}

Table 1: Calculated structure and ban GaAs $(x+y \leq 10)$

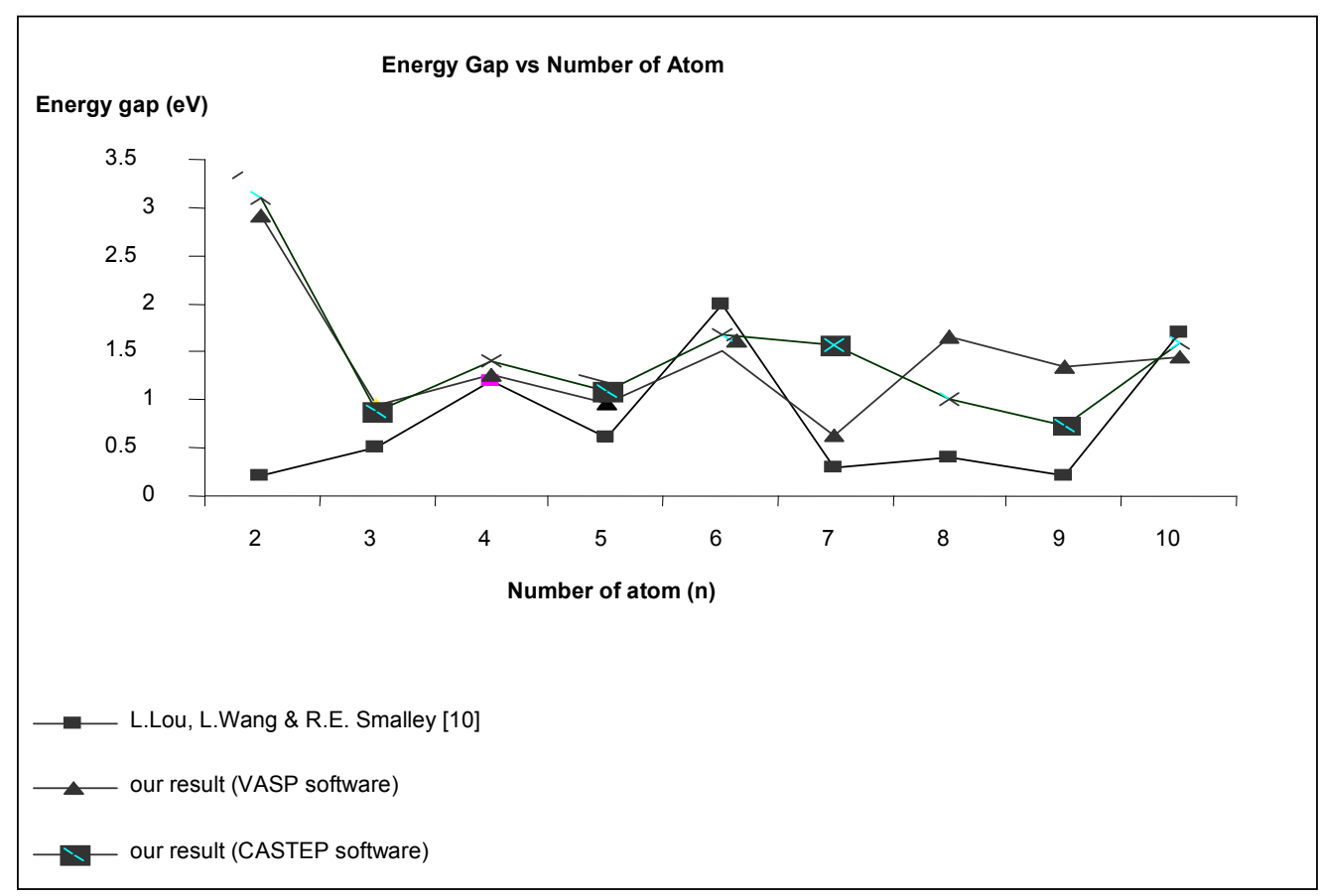

Figure 3: Bandgap (HOMO-LUMO) for $\mathrm{Ga}_{x} \mathrm{As}_{y}$ gap for each 


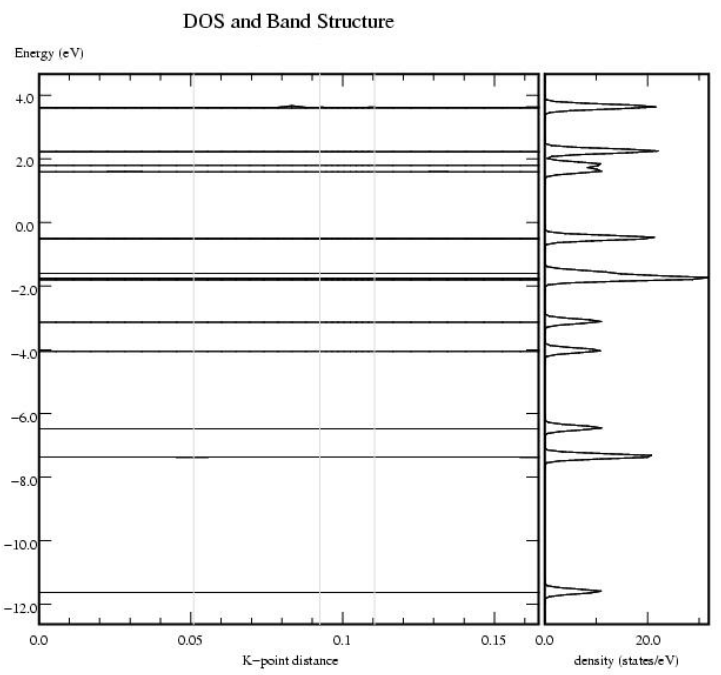

Figure 4: (a)Typical bandstructure of GaAs

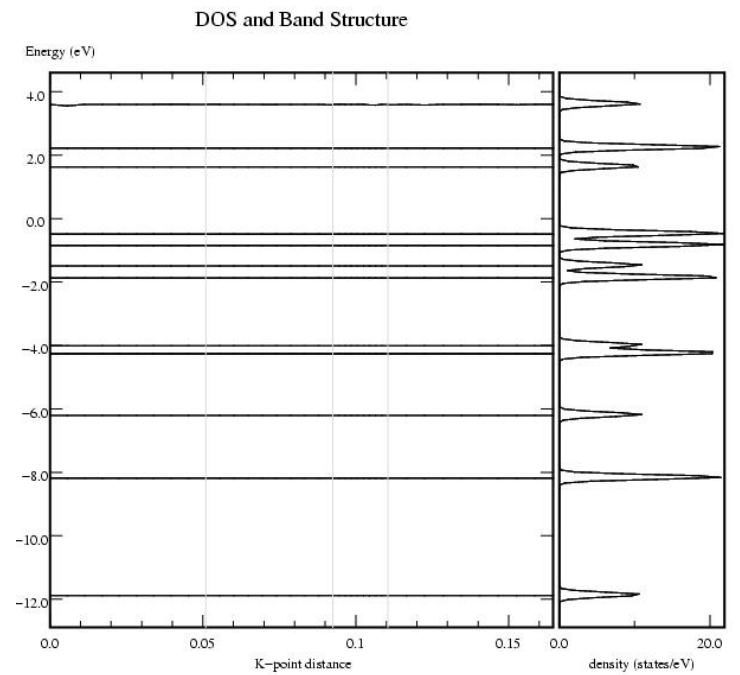

(b) Bandstructure of $\mathrm{Ga}_{3} \mathrm{As}_{3}$

\section{Conclusion}

Calculation using DFT and LDA shows bandgap energies of gallium arsenide clusters are larger than bulk gallium arsenide. It shows that electronic properties of nanocluster are differing from the bulk where bandgap widening is observed. Bandgap values change between $0.7370 \mathrm{eV}$ to $3.0956 \mathrm{eV}$. It depends strongly on the number of atoms and atomic structure.

\section{Acknowledgement}

We would like to thanks for the Ministry of Science, Technology and Innovation Malaysia for financial support through IRPA funding 09-02-06-0159-SR0013/06-05.

\section{References}

[1] Jinwoo Cheon, Young-wook Jun and Sang-Min Lee (2004); Architecture of Nanocrystal Building Blocks, In Vincent Rotello, Nanoparticles: building blocks for nanotechnology (Chap 2, pg 53-87), Kluwer Academic.

[2] Y. Wang and J. P. Pedew (1991); Phys. Rev. B 44, 13298.

[3] G. Kreese and D. Joubert (1999); Phys, Rev. B 59, 1758.

[4] M. P. Teter, M. C. Payne (1995); Phys. Rev. B 51, 4041.

[5] S. F. Li and X. G. Gong (2005); J. Chem. Phy., 122, 174311.

[6] G. Kresse and J. Furthmüller, Phys.Rev. B 54, 11169 (1996)

[7] M.P. Teter, M. C. Payne, and D.C. Allan, Phys. Rev. B 40, 12255 (1995)

[8] L.Lou, P. Nordlander and R. E. Smalley; J. Chem. Phys, Vol 97 (1992)

[9] K M Song, A K Ray and P K Khowash ; J Phys. B : At .Mol Opt. Phys 27 (1994) 1637 - 1648 